\title{
Acute myocarditis: aetiology, diagnosis and management
}

\author{
Authors: Temi Lampejo, ${ }^{A}$ Simon M Durkin, ${ }^{B}$ Naman Bhatt ${ }^{C}$ and Oliver Guttmann ${ }^{D}$
}

Acute myocarditis is a serious, likely underdiagnosed condition affecting people of all ages and for which the number of UK hospital admissions is rising. A primary diagnosis of myocarditis accounted for $0.04 \%$ (36.5 per 100,000) of all hospital admissions in England between 1998 and 2017, although this is likely to be an underestimate of the true burden of myocarditis. The aetiology is broad, including infective and inflammatory conditions as well as exposure to toxic agents. Clinical features are varied and overlap with other acute cardiac conditions making diagnosis a challenge. Cardiovascular magnetic resonance imaging currently serves as the gold standard non-invasive diagnostic modality. If an underlying aetiological process is identified, then therapy may be directed at the cause; however, for most, treatment is supportive and aimed at managing any complications such as heart failure or arrhythmias. There is emerging evidence for immunosuppressive therapy in certain cases. Prognosis is generally good with recovery in most; however, up to $30 \%$ with biopsy-proven myocarditis progress to develop a dilated cardiomyopathy and its potential associated complications. All-cause mortality in the UK for patients presenting to hospital with acute myocarditis is approximately $4 \%$.

KEYWORDS: myocarditis, myopericarditis, chest pain, cardiac inflammation

DOI: 10.7861/clinmed.2021-0121

\section{Introduction}

The myocardium, the middle layer of the heart, contains cardiac muscle cells (cardiac myocytes) enabling the heart to contract. The myocardium is lined internally by the endocardium and externally by the epicardium. Myocarditis is an inflammatory condition of the myocardium which may occur secondary to an infection, may be immune-mediated or can occur due to exposure

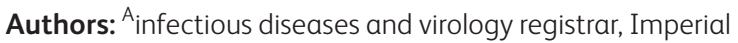
College Healthcare NHS Trust, London, UK; ${ }^{B}$ clinical fellow in infectious diseases, Imperial College Healthcare NHS Trust, London, UK; C radiology registrar, London North West University Healthcare NHS Trust, London, UK; ${ }^{\mathrm{D}}$ consultant cardiologist and honorary associate professor, Barts Health NHS Trust, London, UK to toxic substances. The Global Burden of Disease study reported an incidence of 22 cases of myocarditis per 100,000 patients based on International Classification of Diseases (ICD) codes on hospital discharge documentation between 1990 and $2013 .{ }^{1}$ Data on all NHS England hospital admissions due to myocarditis between 1998 and 2017 showed that over the 19-year period there were 12,927 admissions with a primary diagnosis of myocarditis accounting for $0.04 \%(36.5$ per 100,000$)$ of all NHS admissions (although this condition is likely underdiagnosed). ${ }^{2}$ Approximately two-thirds of patients were men and the median age was 33 years in men compared to 46 years in women. The median length of hospital admission was 4.2 days. There was an $88 \%$ increase in admissions with myocarditis over the study period (compared to a $57 \%$ increase in all cardiology admissions) indicating an increasing burden of hospitalisation due to myocarditis in recent years. An expert consensus group has published a consensus document on the management of acute myocarditis and their key messages are included in this review. ${ }^{3}$

\section{Definition and classification of the various forms of myocarditis}

Myocarditis may present in acute, fulminant, subacute and chronic forms. Acute myocarditis can be defined as a period of $<1$ month between symptom onset and diagnosis. ${ }^{3}$ Fulminant myocarditis is a severe, rapidly evolving form of acute myocarditis with associated cardiogenic shock requiring inotropes or mechanical circulatory support. Subacute myocarditis is characterised by ongoing myocardial damage due to a persistent/recurrent stimulus for myocardial inflammation but can also be defined as a healing myocarditis if there is evidence of a previous active myocarditis. Additionally, subacute myocarditis can be defined as a period of $>1$ to 3 months between symptom onset and diagnosis. When symptoms continue for a prolonged period ( $>1$ month), the disease process is considered to be a chronic inflammatory cardiomyopathy (thus there is an overlap with the definition of subacute myocarditis).

Histopathologically, myocarditis is characterised by an inflammatory cellular infiltrate (which may be focal or diffuse) in the presence or absence of cardiac myocyte injury. ${ }^{4}$ The type of cellular infiltrate can be used to classify myocarditis: ${ }^{3}$

> Lymphocytic myocarditis - associated with a range of pathogens (predominantly viruses), drugs, radiation exposure and autoimmune disorders.

> Eosinophilic myocarditis - a relatively uncommon form associated with parasitic infections, hypersensitivity reactions 
to various agents, eosinophilic inflammatory disorders and rarely a neoplastic process.

> Giant-cell myocarditis - idiopathic in most cases but can also be associated with certain autoimmune disorders including autoimmune thyroid disease and inflammatory bowel diseases. > Granulomatous myocarditis - associated with sarcoidosis.

\section{Clinical presentation}

Myocarditis, which affects people of all ages (symptom onset typically between 20 and 50 years of age), has a wide range of clinical presentations and therefore its diagnosis can be challenging. ${ }^{5}$ Although it may be asymptomatic, the most common presenting features are chest pain (in $85-95 \%$ of cases), fever (in approximately $65 \%$ ) and dyspnoea (19-49\% of cases). ${ }^{3}$ Acute myocarditis therefore needs to be differentiated from acute coronary syndromes and acute pericarditis given the overlapping clinical features. Of additional importance is a detailed family history aiming to exclude any underlying inherited cardiomyopathies, for example, left-dominant arrhythmogenic cardiomyopathy, ${ }^{6}$ which may present with or be misdiagnosed as acute myocarditis alone. Other presenting features of myocarditis include palpitations, syncope and fatigue. Up to $80 \%$ of patients with acute myocarditis have prodromal symptoms; therefore patients may have a history of a preceding flu-like, respiratory and/or gastrointestinal illness. On examination, there may be no significant findings, however, as $26 \%$ of patients with acute myocarditis present with complicating features, that is, left ventricular systolic dysfunction, sustained ventricular arrhythmias or a fulminant presentation including a low cardiac output syndrome; ${ }^{7}$ there may be features consistent with heart failure, cardiogenic shock or even cardiac arrest. Fulminant presentations of myocarditis need to be differentiated from other conditions that may cause haemodynamic instability in association with acute myocardial dysfunction (eg septic shock). ${ }^{3}$

\section{Key investigations}

The ECG, generally the first investigation performed, is often abnormal and most commonly shows ST segment elevation (often in the inferior and lateral leads), as may be seen in ST-elevation myocardial infarction (STEMI). Other ECG findings include bradycardia, tachycardia, atrioventricular block or ventricular arrhythmias. ${ }^{8}$ The full blood count may be normal, although the presence of a raised eosinophil count may be suggestive of an eosinophilic myocarditis (prompting further investigation into the cause of the eosinophilia). The C-reactive protein (CRP) is elevated in over $80 \%$ of patients and the erythrocyte sedimentation rate (ESR) is also frequently elevated (which if remains persistently high may suggest an autoimmune aetiology). ${ }^{9}$ Plasma cardiac biomarkers, such as troponins, may be elevated but a normal troponin does not rule out myocarditis. ${ }^{10}$ Measurement of serum brain natriuretic peptide (BNP) levels are also generally recommended but again a normal value does not exclude myocarditis. Very high levels of cardiac biomarkers (eg troponin and BNP) may, however, prompt more urgent timing of further investigations (such as cardiovascular magnetic resonance imaging (CMR)/endomyocardial biopsy).

The symptoms and signs of acute myocarditis overlap considerably with those of pericarditis, although both can and frequently do occur concomitantly, that is, myopericarditis. ST segment elevation can be seen on the ECG in both myocarditis and pericarditis. The ST elevation tends to be more widespread with pericarditis but ECG features alone are insufficient to differentiate. ${ }^{11}$ An elevated troponin is more suggestive of myocarditis or myopericarditis than pericarditis alone.

Transthoracic echocardiography (TTE) forms a routine part of the investigation of suspected myocarditis; however, there are a broad range of possible findings in acute myocarditis (which may also be seen in other cardiac conditions). Possible echocardiographic findings include a reduced left ventricular ejection fraction (LVEF), diastolic dysfunction, increased cardiac wall thickness, segmental wall motion abnormalities, abnormal echogenicity of the myocardium or a pericardial effusion (if there is coexistent pericardial involvement). ${ }^{3,12}$ Cardiovascular magnetic resonance imaging with intravenous gadolinium-based contrast agents is recommended in patients with suspected acute myocarditis and should certainly be performed in individuals with more severe/fulminant forms (provided the patient is stable enough to undergo the scan). Cardiovascular magnetic resonance imaging serves as the reference standard non-invasive modality to determine the presence or absence of myocardial inflammation and other associated abnormalities., ${ }^{3,13}$ CMR may have reduced accuracy within the first few days of illness but should be performed within 2 to 3 weeks of symptom onset. CMR can also be useful for monitoring disease progression/activity. ${ }^{14}$

Endomyocardial biopsy has traditionally been the reference standard diagnostic test for myocarditis (with specimens typically sent for histology, immunohistochemistry and polymerase chain reaction (PCR) for potential infectious agents). ${ }^{15}$ It is useful for diagnostic confirmation and in determining the aetiopathogenic form of myocarditis (eg giant-cell myocarditis, lymphocytic or sarcoid). An endomyocardial biopsy should be performed in a high-volume centre with experience in the management of myocarditis as it is an invasive procedure with associated potential risks, albeit lower with experienced teams. There is up to a $9 \%$ risk of cardiac complications in low-volume settings and also a risk of other complications including peripheral vascular complications, arrhythmias, pneumothoraces and post-procedure infectious complications. ${ }^{16}$ Some patients with suspected myocarditis are considered unsuitable for endomyocardial biopsy. Additionally, myocardial inflammation is often patchy and therefore a biopsy may be non-diagnostic if unaffected tissue is biopsied. Endomyocardial biopsy is most commonly performed by a transfemoral approach; however, a transradial approach is also possible and has a similar complication profile. ${ }^{17}$ There is increasing reliance on CMR for the diagnosis of myocarditis; however, endomyocardial biopsy may be performed in specific cases when it is deemed likely to significantly impact upon patient management. The indications for endomyocardial biopsy in the setting of acute myocarditis and chronic inflammatory cardiomyopathy as per the European Society of Cardiology-Heart Failure Association (ESC-HFA) and American College of Cardiology Foundation/American Heart Association (ACCF/AHA) guidelines are as follows ${ }^{18,19}$ (although one should be aware that there are several other indications for endomyocardial biopsy in the investigation of other cardiac disorders ${ }^{20}$ ):

$>$ New-onset heart failure ( $<2$ weeks) with a normal or dilated left ventricle and haemodynamic compromise or within 2 weeks to 3 months if associated with ventricular arrhythmias, high-grade 
Box 1. Investigations recommended in all patients with suspected myocarditis

\section{Laboratory investigations}

Full blood count (an eosinophilia may be suggestive of an eosinophilic myocarditis)

Liver function tests

Renal function and electrolytes

CRP and ESR (an elevated ESR in particular may suggest an underlying inflammatory condition)

Plasma troponin/CK

Serum BNP

\section{Imaging/Other investigations}

Chest X-ray

Electrocardiography

Transthoracic echocardiography

CMR with a gadolinium-based contrast agent (except if a patient is too unstable or in select patients with an implantable device in whom CMR may be contraindicated)

$\mathrm{BNP}=$ brain natriuretic peptide; $\mathrm{CK}=$ creatine kinase; $\mathrm{CMR}=$ cardiovascular magnetic resonance imaging; $C R P=C$-reactive protein; $E S R=$ erythrocyte sedimentation rate.

AV block (second or third degree) or failure to respond to usual care within 1 to 2 weeks.

$>$ Acute myocarditis or suspected chronic inflammatory cardiomyopathy associated with peripheral eosinophilia.

> Acute myocarditis or chronic inflammatory cardiomyopathy with persistent or relapsing release of biomarkers of myocardial necrosis, particularly if associated to an autoimmune disorder or ventricular arrhythmias or high-degree atrioventricular block.

$>$ Myocarditis in the setting of immune checkpoint inhibitor therapy, where appropriate diagnosis has implications for patients receiving additional cancer therapy.

A fluorodeoxyglucose positron emission tomography (FDGPET) scan is also capable of assessing myocarditis in view of increased myocardial FDG uptake in the setting of myocardial inflammation. ${ }^{21}$ Fluorodeoxyglucose positron emission tomography is not routinely performed but may be used in certain instances such as when CMR findings are inconclusive or when extra-cardiac inflammation is suspected such as in sarcoidosis. Box 1 summarises the key investigations that should be performed in all patients with suspected acute myocarditis. Additional investigations, as discussed in the next section of this review (and summarised in Table 1), will also need further consideration depending on the most likely aetiologies.

\section{Further investigation into the aetiology of myocarditis}

The history may provide important clues to the potential aetiology and help guide further investigations. Viral infections are the most commonly identified cause of myocarditis, in particular coxsackieviruses, adenoviruses and parvovirus B19. ${ }^{22}$ In recent months, cardiac inflammation and acute/fulminant myocarditis has become a well-recognised complication of severe acute respiratory syndrome coronavirus 2 (SARS-CoV-2) infection. ${ }^{23}$ Obtaining a travel history is important as travel-associated infections such as dengue ${ }^{24}$ and Zika virus ${ }^{25}$ have shown association with

Table 1. Additional investigations to consider in patients with suspected myocarditis

\section{Investigation}

Thyroid function tests

Serum angiotensin converting enzyme level

Drug screen

Plasma and 24-hour urine metanephrines

Nasopharyngeal swab for respiratory viruses PCR including influenza, SARS-CoV-2, adenoviruses and enteroviruses ASO titre and throat swab for bacterial culture Testing for Mycoplasma pneumoniae (by PCR \pm serology) Blood borne virus screen, ie HIV, HBV and HCV serology

Blood for EBV, CMV and parvovirus B19 serology Autoantibodies (ANA initially and then possible further autoantibody screening guided by the clinical picture) Urine analysis with urinary sediment (to look for casts/ crystals/cells)

Borrelia serology

FDG-PET

Endomyocardial biopsy
Particular instance(s) to consider the investigation (although testing should not necessarily be limited to these specific situations)

Clinical features suggestive of hyperthyroidism

Suspicion of sarcoidosis

Suspicion of myocarditis secondary to illicit drug use

Fulminant myocarditis/cardiogenic shock (phaeochromocytoma can cause a catecholamine-mediated myocarditis)

History of a possible recent viral illness

Possible current/recent streptococcal infection

Current/recent atypical pneumonia

Identifiable risk factors, eg injecting drug use and/or abnormal liver function tests

Recent glandular fever-like illness and/or rash

Suspicion and/or family history of an autoimmune/inflammatory condition

Abnormal renal function and/or suspicion of an inflammatory condition with renal involvement, eg lupus nephritis

Suspicion of Lyme disease, eg recent erythema migrans rash/recent tick bite/recent visit to a forest/grassy area

When CMR is contraindicated or inconclusive and in suspected sarcoidosis (to detect active myocardial inflammation/potentially identify extracardiac features of sarcoidosis/identify sites amenable to biopsy) Specific cases where deemed by a cardiologist/multi-disciplinary team likely to impact on patient management

ANA = antinuclear antibody; $\mathrm{ASO}=$ antistreptolysin $\mathrm{O} ; \mathrm{CMR}=$ cardiovascular magnetic resonance imaging; $\mathrm{CMV}=$ cytomegalovirus; EBV = Epstein-Barr virus; FDG-PET = fluorodeoxyglucose-positron emission tomography; HBV = hepatitis B virus; $\mathrm{HCV}=$ hepatitis $\mathrm{C}$ virus; $\mathrm{PCR}=$ polymerase chain reaction; $\mathrm{SARS}$-CoV-2 = severe acute respiratory syndrome coronavirus 2. 
myocarditis. A detailed drug history (including prescription, over-the-counter and recreational drugs) and recent vaccination history are vital. The presence of immunosuppression may broaden the differential further and certain aetiological agents (such as Epstein-Barr virus, cytomegalovirus and fungi) that are typically deemed to be responsible for a relatively low disease burden in the immunocompetent may need to be given greater consideration in an immunosuppressed patient. Additionally, establishing the possible presence of an autoimmune/inflammatory condition (eg systemic lupus erythematosus and eosinophilic granulomatosis with polyangiitis) is important given the observation that 7\% of patients with myocarditis have an associated autoimmune disorder ${ }^{7}$ and these conditions may be amenable to additional therapeutic interventions. Features suggestive of a potential autoimmune/inflammatory condition include a history of fevers, fatigue, musculoskeletal symptoms, lymphadenopathy, rashes, hair loss, mucosal ulceration, neuropathic pain, ocular manifestations (eg uveitis), altered bowel habit, impaired renal function, and peripheral blood eosinophilia as well as a possible family history of an autoimmune/inflammatory disease. Sarcoidosis, a multisystem granulomatous disease, may present with several of these features but can also be asymptomatic. When considering sarcoidosis, extra-cardiac features to assess for the presence of sarcoidosis include erythema nodosum, respiratory manifestations (such as cough and dyspnoea) and bilateral hilar adenopathy (although these features are not specific to sarcoidosis). However, sarcoidosis can also present predominantly with cardiac manifestations (clinically manifest cardiac involvement occurs in $5 \%$ of patients with pulmonary/systemic sarcoidosis). ${ }^{5,26}$

There is a wide array of potential aetiological triggers for acute myocarditis, which are summarised in Box 2. It is not

\section{Box 2. Causes of myocarditis}

\section{Infectious causes}

Viral, eg adenoviruses, enteroviruses (including coxsackieviruses), parvovirus B19, influenza, severe acute respiratory syndrome coronavirus 2 (SARS-CoV-2) and HIV-1

Bacterial, eg Mycoplasma pneumoniae, Spirochaetes (including Treponema pallidum and Borrelia burgdorferi), Staphylococcus spp. and Streptococcus spp.

Fungal, eg Aspergillus spp. and Candida spp.

Protozoal, eg Plasmodium spp. and Toxoplasma spp.

Helminth, eg Schistosoma spp. and Toxocara spp.

Rickettsial (and Rickettsia-like organisms), eg Rickettsia rickettsiae and Coxiella burnetii (a Rickettsia-like organism)

\section{Non-infectious causes}

Hypersensitivity/Allergic, eg antibiotics, anticonvulsants and various vaccines

Systemic inflammatory disorders, eg sarcoidosis, systemic lupus erythematosus, thyrotoxicosis (eg Graves' disease), diabetes mellitus (insulin dependent), inflammatory bowel disease and granulomatosis with polyangiitis

Toxic myocarditis, eg amphetamines, cocaine, bites/stings, catecholamines, radiation, chemotherapy and immune checkpoint inhibitors

Transplant rejection, eg heart transplantation and stem cell transplantation

Autoreactive, eg giant-cell myocarditis and lymphocytic myocarditis recommended or practical to test for all the potential causes and therefore the specific further investigations performed, as summarised in Table 1, will be guided primarily by a detailed history and thorough examination as well as the preliminary investigation findings.

Concomitant coronary artery disease should be considered in all patients presenting with suspected acute myocarditis and should be excluded either indirectly (on the basis of a CMR showing a non-ischaemic pattern of late enhancement) or directly with either of a computed tomography (CT) cardiac coronary angiogram or an invasive coronary angiogram.

\section{Treatment}

Multispecialty input (cardiology, infectious diseases, rheumatology, immunology and/or respiratory medicine) is often required to determine the aetiology and direct management in cases of suspected myocarditis. If a specific cause is identified, treatment (where available) is directed at the underlying aetiological process/ condition such as anti-infective agents in cases of treatable infection or corticosteroids/other immunosuppressants in cases of immunemediated disease. The strongest evidence for immunosuppressive therapy is in cardiac sarcoidosis, giant-cell myocarditis and also in autoimmune rheumatic disease. ${ }^{27,28}$ The aetiology, is however, often not identified or is commonly of a viral aetiology for which there is no specific treatment and/or specific treatment is not required. Treatment of these cases is usually supportive with the management of any associated complications such as arrhythmias or heart failure and severe cases may require intensive care unit (ICU) admission. There is emerging evidence suggesting a potential benefit of selective immunosuppressive therapy in chronic myocarditis patients, ${ }^{29}$ however, this is currently not routine and requires multidisciplinary team discussion prior to initiation. In rare instances, empirical immunosuppression may be trialled in critically ill patients who are too unwell to undergo an endomyocardial biopsy, particularly where giant-cell myocarditis is suspected.

Non-steroidal anti-inflammatory drugs (NSAIDs), which are a mainstay of therapy in pericarditis, are not recommended in myocarditis, partly due to their ineffectiveness and potential exacerbation of myocarditis in animal models ${ }^{30}$ but also due to the fact that NSAIDs should be generally avoided in heart failure in view of the potential risk of worsening of heart failure. ${ }^{31}$

Patients with significant haemodynamic compromise/fulminant myocarditis may require inotropic support and, in some cases, use of a temporary mechanical circulatory support device such as venoarterial extracorporeal membrane oxygenation (VA-ECMO) and rotary blood pumps. ${ }^{3}$ Early ICU review and admission for pharmacological inotropic and/or anti-arrhythmic treatment or mechanical support may be necessary. Support should be considered and implemented early. A minority of patients may not be stabilised by inotropic support or mechanical circulatory support (or may be unable to be weaned off such devices). Myocarditis patients with heart failure or arrhythmias refractory to these temporary strategies may be considered for cardiac transplantation or left ventricular assist device implantation. Such patients should be promptly discussed with a cardiac transplant centre.

\section{Follow-up, prognosis and complications}

Most patients with acute myocarditis recover and myocardial inflammation spontaneously resolves without specific treatment. 
Falling inflammatory markers, declining cardiac biomarker levels, improvement of cardiac function and absence of arrhythmia are indicators of clinical improvement and serve as useful monitoring tools. Within 3 months of discharge, patients should be followed up by a consultant cardiologist, ideally in a dedicated clinic; the timing is dependent on their clinical condition at discharge. Following a diagnosis of myocarditis, refraining from participation in competitive sports/vigorous exercise is recommended for 3-6 months (to reduce risk of cardiac remodelling and sudden cardiac death). ${ }^{32}$

On baseline CMR at the time of diagnosis, the presence and anteroseptal location of mid-wall late gadolinium enhancement ${ }^{33}$ and low baseline LVEF have been demonstrated to be important CMR predictors of adverse cardiovascular events in acute myocarditis. ${ }^{3}$ Repeat CMR is useful in the follow-up of acute myocarditis and may be performed (typically at 6-12 months after the index event but may be sooner depending on the patient's clinical status) ${ }^{3}$ particularly if there is concern regarding an ongoing active disease process. If evidence of ongoing myocardial inflammation is present, a subsequent endomyocardial biopsy may be performed with a view to potentially guide further specific treatment.

Recurrence of acute myocarditis is rare, with $1.1 \%$ of patients diagnosed with acute myocarditis reporting a history of previous myocarditis. ${ }^{7}$ However, up to $30 \%$ of cases of biopsyproven myocarditis progress to dilated cardiomyopathy, ${ }^{10}$ particularly in individuals with significantly impaired LVEF, ${ }^{34}$ and LVEF at the time of hospital admission has been shown in some studies to be a useful prognostic marker. ${ }^{8,35}$ UK NHS data for patients admitted with myocarditis indicate an all-cause mortality of approximately $4 \%$ with $20 \%$ of these deaths attributable to non-ischaemic dilated cardiomyopathy. ${ }^{2}$ The prognosis of myocarditis can, however, vary widely depending on the aetiology/classification with the giantcell form traditionally exhibiting the poorest prognosis; an approximately $90 \%$ rate of death or cardiac transplantation and a median survival of 5.5 months from symptom onset was previously associated with this form of myocarditis. ${ }^{36}$ However, outcomes have significantly improved in recent years with the use of immunosuppressive therapy and there is now a significantly reduced need for mechanical support and/or cardiac transplantation in patients with giant-cell myocarditis. ${ }^{37}$ These interventions are still required in certain cases and postcardiac transplant survival for giant-cell myocarditis is $94 \%$ at 1 year, $82 \%$ at 5 years and $68 \%$ at 10 years (similar to other aetiologies of myocarditis). ${ }^{37}$ However, $20-25 \%$ of patients develop a recurrence of giant-cell myocarditis even after cardiac transplantation (which is often asymptomatic and detected on surveillance endomyocardial biopsy) thus typically requiring further immunosuppression. ${ }^{37}$

\section{Conclusion}

The disease burden of acute myocarditis is rising in the UK. There is a wide aetiological spectrum and, in many cases, the underlying cause is not identified. Clinical features and initial investigations may be suggestive of myocarditis; however, many of the presenting features overlap with other cardiac conditions. Endomyocardial biopsy has traditionally been the reference standard diagnostic test and CMR remains a crucial noninvasive diagnostic method. Treatment is generally supportive and aimed at managing complications. Some patients require immunosuppressive therapies. In cases where an aetiological trigger is identified, specific therapy may be appropriate in certain instances. Overall prognosis from myocarditis is good and the majority recover; however, a minority of patients develop longterm sequelae often in the form of a dilated cardiomyopathy further complicated by heart failure and/or arrhythmias.

\section{Key practice points}

> Acute myocarditis should be considered in patients of all ages with any of the following:

Symptoms including chest pain, fever or breathlessness

Palpitations (arrhythmia)

Syncope or cardiac arrest

A prodromal illness

Electrocardiographic (ECG) changes, eg ST segment elevation Elevated cardiac biomarkers (eg troponin)

Regional or global contractile dysfunction of the heart

> A detailed history and thorough examination are key to guiding further investigations for a potential aetiology, with particular consideration given to any recent/current infections (especially a recent viral illness), autoimmune/inflammatory conditions and exposure to potentially toxic substances (eg certain drugs).

> Myocarditis is defined as an inflammatory disease of the cardiac muscle, diagnosed on endomyocardial biopsy by established histological, immunological and immunohistochemical criteria.

> Cardiovascular magnetic resonance imaging serves as the reference standard non-invasive test for the diagnosis of myocarditis.

$>$ Unless a specific cause is identified for which treatment is available and required, acute myocarditis treatment is generally supportive and focuses on the management of potentially associated complications such as heart failure and arrhythmias.

> Non-steroidal anti-inflammatory drugs, unlike with acute pericarditis, are not routinely recommended in the management of acute myocarditis.

> Overall prognosis is good (but can vary depending on the type of myocarditis) with most patients recovering from their acute episode without long-term sequelae and with a low risk of recurrence $(\sim 1 \%)$; however, a dilated cardiomyopathy can develop in up to $30 \%$ of individuals with biopsy-proven myocarditis. .

\section{References}

1 Global Burden of Disease Study 2013 Collaborators. Global, regional, and national incidence, prevalence, and years lived with disability for 301 acute and chronic diseases and injuries in 188 countries, 1990-2013: a systematic analysis for the Global Burden of Disease Study 2013. Lancet 2015;386:743-800

2 Lota AS, Halliday B, Tayal U et al. Epidemiological trends and outcomes of acute myocarditis in the National Health Service of England. Circulation 2019;140:A11463. https://www.ahajournals. org/doi/10.1161/circ.140.suppl_1.11463

3 Ammirati E, Frigerio M, Adler ED et al. Management of acute myocarditis and chronic inflammatory cardiomyopathy: an expert consensus document. Circ Heart Fail 2020;663-87.

4 Aretz HT, Billingham ME, Edwards WD et al. Myocarditis. A histopathologic definition and classification. Am J Cardiovasc Pathol 1987;1:3-14.

5 Caforio ALP, Calabrese F, Angelini A et al. A prospective study of biopsy-proven myocarditis: prognostic relevance of clinical and aetiopathogenetic features at diagnosis. Eur Heart J 2007;28:1326-33. 
6 Sen-Chowdhry S, Syrris P, Prasad SK et al. Left-dominant arrhythmogenic cardiomyopathy. An under-recognized clinical entity. J Am Coll Cardiol 2008;52:2175-87.

7 Ammirati E, Cipriani M, Moro C et al. Clinical presentation and outcome in a contemporary cohort of patients with acute myocarditis multicenter Lombardy registry. Circulation 2018;138:1088-99.

8 Younis A, Matetzky S, Mulla W et al. Epidemiology characteristics and outcome of patients with clinically diagnosed acute myocarditis. Am J Med 2020;133:492-9.

9 Aquaro GD, Perfetti M, Camastra G et al. Cardiac MR with late gadolinium enhancement in acute myocarditis with preserved systolic function: ITAMY study. J Am Coll Cardiol 2017;70:1977-87.

10 Caforio ALP, Pankuweit S, Arbustini E et al. Current state of knowledge on aetiology, diagnosis, management, and therapy of myocarditis: a position statement of the European Society of Cardiology Working Group on Myocardial and Pericardial Diseases. Eur Heart ] 2013;34:2636-48.

11 Ismail TF. Acute pericarditis: update on diagnosis and management. Clin Med (Lond) 2020;20:48-51.

12 Felker GM, Boehmer JP, Hruban RH et al. Echocardiographic findings in fulminant and acute myocarditis. J Am Coll Cardiol 2000;36:227-32

13 Ferreira VM, Schulz-Menger J, Holmvang G et al. Cardiovascular magnetic resonance in nonischemic myocardial inflammation: expert recommendations. J Am Coll Cardiol 2018;72:3158-76.

14 Liguori C, Farina D, Vaccher F, Ferrandino G, Bellini D, Carbone I. Myocarditis: imaging up to date. Radiol Med 2020;125:1124-34.

15 Leone O, Veinot JP, Angelini A et al. 2011 consensus statement on endomyocardial biopsy from the Association for European Cardiovascular Pathology and the Society for Cardiovascular Pathology. Cardiovasc Pathol 2012;21:245-74.

16 Singh V, Mendirichaga R, Savani GT et al. Comparison of utilization trends, indications, and complications of endomyocardial biopsy in native versus donor hearts (from the Nationwide Inpatient Sample 2002 to 2014). Am J Cardiol 2018:121:356-63.

17 Tyler Z, Guttmann OP, Dhinoja M et al. The safety and feasibility of transitioning from transfemoral to transradial access left ventricular endomyocardial biopsy. J Invasive Cardiol 2020;32:E349-54.

18 Ponikowski P, Voors AA, Anker SD et al. 2016 ESC guidelines for the diagnosis and treatment of acute and chronic heart failure: The task force for the diagnosis and treatment of acute and chronic heart failure of the European Society of Cardiology (ESC). Developed with the special contribution of the Heart Failure Association (HFA) of the ESC. Eur J Heart Fail 2016;18:891-975.

19 Yancy CW, Jessup M, Bozkurt B et al. 2013 ACCF/AHA guideline for the management of heart failure: a report of the American College of Cardiology Foundation/American Heart Association Task Force on Practice Guidelines. Circulation 2013;128.

20 Cooper LT, Baughman KL, Feldman AM et al. The role of endomyocardial biopsy in the management of cardiovascular disease: a scientific statement from the American Heart Association, the American College of Cardiology, and the European Society of Cardiology. Circulation 2007;116:2216-33.
21 Chen W, Jeudy J. Assessment of myocarditis: Cardiac MR, PET/CT, or PET/MR? Curr Cardiol Rep 2019;21:76.

22 Cooper LTJr. Medical progress: myocarditis. New Engl J Med 2009; 360:1526-38.

23 Guzik TJ, Mohiddin SA, Dimarco A et al. COVID-19 and the cardiovascular system: implications for risk assessment, diagnosis, and treatment options. Cardiovasc Res 2020;116:1666-87.

24 Bhatt M, Soneja M, Farooqui FA et al. Myocarditis in admitted patients with dengue fever. Infection 2020;48:899-903.

25 Aletti M, Lecoules S, Kanczuga $V$ et al. Transient myocarditis associated with acute Zika virus infection. Clin Infect Dis 2017;64:678-9.

26 Birnie DH, Nery PB, Ha AC, Beanlands RSB. Cardiac sarcoidosis. J Am Coll Cardiol 2016:68:411-21.

27 Schultheiss HP, Khl U, Cooper LT. The management of myocarditis. Eur Heart J 2011;32:2616-25.

28 Cooper LT, Hare JM, Tazelaar HD et al. Usefulness of immunosuppression for giant cell myocarditis. Am J Cardiol 2008;102:1535-9.

29 Merken J, Hazebroek M, Van Paassen P et al. Immunosuppressive therapy improves both short- and long-term prognosis in patients with virus-negative nonfulminant inflammatory cardiomyopathy. Circ Heart Fail 2018;11:e004228.

30 Khatib R, Reyes MP, Smith F, Khatib G, Rezkalla S. Enhancement of coxsackievirus B4 virulence by indomethacin. J Lab Clin Med 1990;116:116-20.

31 Arfè A, Scotti L, Varas-Lorenzo C et al. Non-steroidal anti-inflammatory drugs and risk of heart failure in four European countries: nested case-control study. BMJ 2016;354:i4857.

32 Tschöpe C, Cooper LT, Torre-Amione G, Van Linthout S. Management of myocarditis-related cardiomyopathy in adults. Circ Res 2019;124:1568-83.

33 Georgiopoulos G, Figliozzi S, Sanguineti F et al. Prognostic impact of late gadolinium enhancement by cardiovascular magnetic resonance in myocarditis: a systematic review and meta-analysis. Circ Cardiovasc Imaging 2021;14:e011492.

34 Liu PP, Mason JW. Advances in the understanding of myocarditis. Circulation 2001;104:1076-82.

35 Chareonthaitawee P, Beanlands RS, Chen W et al. Joint SNMMIASNC expert consensus document on the role of 18F-FDG PET/CT in cardiac sarcoid detection and therapy monitoring. J Nucl Cardiol 2017:24:1741-58.

36 Cooper LT, Berry G], Shabetai R. Idiopathic giant-cell myocarditis natural history and treatment. N Engl J Med 1997;336:1860-6.

37 Bang V, Ganatra S, Shah SP et al. Management of patients with giant cell myocarditis: JACC Review topic of the week. J Am Coll Cardiol 2021;77:1122-34.

Address for correspondence: Dr Temi Lampejo, Department of Infection, Hammersmith Hospital, Imperial College Healthcare NHS Trust, Du Cane Road, London W12 0HS, UK. Email: temi.lampejo@nhs.net 